\title{
De las tormentosas relaciones entre forma y contenido en el pensamiento: crónica de un romance anunciado
}

\author{
JUAN IGNACIO POZO * \\ Dpto. de Psicología Básica, Social y Metodología \\ Facultad de Psicología \\ Universidad Autónoma de Madrid
}

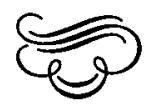

\section{Resumen}

El presente articulo estudia las complejas y cambiantes relaciones entre forma y contenido en la psicologia del pensamiento. Tradicionalmente se han distinguido dos estadios en el razonamiento y la solución de problemas: una fase de representación, centrada básicamente en el contenido del problema, y una segunda fase de proceso o razonamiento propiamente dicho, en la que primarian los aspectos lógico-formales. El artículo analiza la evolución desde los estudios sintäcticos del pensamiento - centrados en las reglas formales que lo rigen-a los estudios semánticos, preocupados sobre todo por el contenido de las representaciones, para acabar proponiendo una integración de ambos aspectos dentro de una visión pragmática del pensamiento

\section{Abstract}

This paper reviews the controversial relationship between form and content in psychological research of thinking. Traditionally, two stages are recognized in reasoning and problem solving: the representation stage, closely linked to the problem content, and the process stage, which is characterized in logical-formal terms. In addition to analyse both the syntactical and semantical models of thinking, respectively concerned with the form and the content of problem, the paper proposes a pragmatic view of thinking, in wich form and content are armoniously integrated and reciprocally influenced.

* El presente artículo es parte de la Tesis Doctoral del autor, dirigida por Mario Carretero, con quien he contraido una deuda interna y, por tanto, impagable. Recuerdo que cuando comencé a trabajar con Mario, hace ya algunos años, el tema de investigación que me propuso para mi Memoria de Licenciatura fue la influencia del contenido en el pensamiento formal. Este artículo, y el trabajo en el que se inscribe, es en parte fruto de aquel impulso iniciático. Pero la deuda excede el contenido y la forma de este artículo. De nuestra estrecha vinculación durante todos estos años he aprendido muchas otras cosas además de las propiamente académicas. Este trabajo se ha beneficiado también de la concesión por la C.A.I.C.Y.T. de la investigación n. $2716 / 83$ sobre sEl desarrollo del pensamiento de la adolescencia a la edad adultax, dirigida por Mario Carretero y Juan A. García Madruga. Las reuniones de dicha investigación han estado dedicadas casi íntegramente a comentar los devaneos amorosos de los que trata este artículo, por lo que tengo que recordar aquí, además de a Mario y a Juan, a Mikel Asensio, Jesús I. Cordero, Javier Recio, Amparo Moreno y María Puy Pérez Echevarría. En el caso de esta última la deuda se remonta tanto en el tiempo, $y$ tan gratamente, que se hace memoria.

Dirección del autor: Juan Ignacio Poz. Dpto. de Psicología Básica, Social y Metodología. Facultad de Psicología. Universidad Autónoma de Madrid. Ciudad Universitaria de Madrid. Ciudad Universitaria de Canto Blanco, 28049 Madrid. 


\section{INTRODUCCION}

Como sucediera con la poesía y otras manifestaciones artísticas en los próximos pero ya remotos tiempos del final del franquismo, la psicología cognitiva sigue viviendo aún una profunda escisión entre forma y contenido, que se manifiesta con especial intensidad en los estudios sobre pensamiento, razonamiento y solución de problemas, pero que trasciende también otras áreas y constituye en sí mismo un problema teórico difícil de resolver para el paradigma cognitivo dominante del procesamiento de información. En aquellos años debatíamos si la poesía, comprometida en el cambio social, debía guiarse por criterios formales o de contenido. Hoy, ocupados en conocer cómo los humanos construyen, manipulan y transforman representaciones sobre el mundo, nos preguntamos si esa representaciones sobre el mundo, nos preguntamos si esas representaciones tienen rasgos exclusivamente formales o también temáticos. Esta polémica es algo más que un debate bizantino. Su solución tiene importantes consecuencias para determinar qué tipo de psicología cognitiva es necesario realizar, de la misma forma que determinaba qué tipo de poesía se acababa escribiendo. Probablemente no sea una casualidad que en ambos casos hayamos acabado confluyendo en una misma solución. Un día descubrimos que la forma y el contenido de un poema deben hallarse en mutua armonía. Determinadas estrofas o formas poéticas se ajustan más dócilmente a determinados temas. Igualmente, ahora estamos descubriendo que el razonamiento tiene forma y contenido al mismo tiempo, pero que ambas no son variables independientes, que puedan manipularse a discreción, sino que interactúan de formas complejas y que nos son en gran medida desconocidas.

En el presente artículo, pretendemos analizar las relaciones entre forma y contenido en el marco de la psicología del pensamiento, mostrando cómo éste no puede reducirse ni a las reglas formales y sintácticas que lo rigen ni a su contenido o semántica, sino que es necesario postular una interacción entre ambos aspectos que atienda a consideraciones pragmáticas y no exclusivamente sintácticas o semánticas. Para ello realizaremos una revisión, no exhaustiva sino indicativa, de los estudios sobre la influencia del contenido en el uso de reglas formales de razonamiento. También nos ocuparemos brevemente, a la inversa, de la influencia del razonamiento sobre los contenidos de memoria o representaciones de los sujetos. Antes de revisar esa literatura es necesario repasar, siquiera someramente, la evolución histórica de las relaciones entre forma y contenido en la psicología del pensamiento.

\section{RELACIONES ENTRE FORMA Y CONTENIDO EN LA PSICOLOGIA DEL PENSAMIENTO}

Tradicionalmente en la psicología del pensamiento se han venido distinguiendo dos componentes diferenciados en el razonamiento o la solución de un problema: la representación que del mismo se hace el sujeto y las operaciones o transformaciones mentales que el sujeto realiza sobre esas representaciones. De hecho, la mayor parte de los modelos elaborados en este área de la psicología cognitiva son modelos duales, al aceptar la existencia de estos dos componentes separados. Muchas veces esta separación, al amparo de la serialidad defendida por el procesamiento de información, es no sólo conceptual sino también tem- 
poral, al postularse modelos secuenciales, en los que existen dos fases sucesivas: una fase inicial de representación y una fase posterior de operación o razonamiento propiamente dicho. Aunque existen posiciones diversas al respecto, en los modelos duales suele considerarse que las operaciones se articulan según criterios formales o sintácticos (o, sin se prefiere así, lógicos) mientras que el contenido o temática del problema se recoge en la representación que del mismo se hace del sujeto.

Pero si todos los modelos duales comparten estos supuestos comunes, existen sin embargo diferencias entre ellos en cuanto a la importancia relativa que conceden a cada uno de los componentes del pensamiento. De hecho, las relaciones de primacía entre ambos han variado considerablemente en los últimos años, poniendo en duda algunos de los principios bàsicos sobre los que se apoyaba tradicionalmente no sólo la psicología del pensamiento sino, en un sentido más general, la propia psicología cognitiva. Esta evolución podemos recogerla agrupando los modelos en dos grandes grupos según su consideración de las relaciones entre forma y contenido (véase más extensamente Pozo, 1987):

a) modelos lógicos, según los cuales razonar consite básicamente en aplicar una serie de reglas formales de carácter general. Según estos modelos el razonamiento se rige por leyes ajenas al contenido de la tarea, que suelen corresponderse con las leyes de la lógica formal. Dentro de este apartado podríamos incluir todos aquellos modelos o teorías de propósitos generales que minimizan la importancia de los contenidos concretos, y reducen el pensamiento o la manipulación de representaciones a sus aspectos sintácticos. Entre esos modelos estarían el Solucionador General de Problemas de Newell y Simon (1972), la teoría de los estadios de Piaget (Piaget, 1970a; Inhelder y Piaget, 1955) y en general todas aquellas posiciones que, desde el procesamiento de información, respetan el logicismo como uno de los postulados básicos de la psicología cognitiva (véase De Vega, 1981). Una versión radical de estos modelos defendería que el uso de esas reglas es plenamente independiente del contenido de las tareas a las que se aplican. Esa sería la posición piagetiana clásica con respecto a las operaciones formales (Inhelder y Piaget, 1955). Sin embargo, versiones más moderadas del mismo planteamiento - a las que acabaría adhiriéndose el propio Piaget (1970b) - reconocen la existencia de «distorsiones factuales» en la aplicación de esas reglas como consecuencia de interpretaciones previas erróneas del contenido del problema (Henle, 1962). Así, el contenido influiría en la solución del problema pero sólo como un sesgo de representación previo al propio proceso de razonamiento, que, en sí mismo, podría seguir siendo explicado mediante criterios formales.

Otros autores consideran sin embargo que las reglas del pensamiento no se ajustan a la lógica formal sino que poseen una slógica» distinta, por lo que dentro de este mismo grupo podrían incluirse también los modelos ilógicos (por ej., Wason y Johnson-Laird, 1972), centrados también en los aspectos formales del pensamiento, y los modelos de lógica natural (Braine, 1978; Braine y Rumaine, 1983).

b) modelos de memoria (o no lógicos), según los cuales los sujetos no utilizan reglas formales de razonamiento sino que se limitan a recuperar de su memoria información relevante para la solución del problema, relacionada con el contenido específico de la tarea. No existirían por tanto unas reglas generales para solucionar problemas, sino contenidos específicos que, aplicados a situaciones concretas, permiten resolverlas, o sea, comprenderlas reduciéndolas a tér- 
minos conocidos (Rumelhart, 1980). De esta forma el pensamiento se reduce por completo a sus aspectos semánticos. Al igual que los modelos lógicos, estos modelos tienen una amplia tradición, con precedentes tales como el «efecto atmósferas postulado por Woodworth y Sells (1935) para los silogismos categóricos o la literatura conductista sobre la solución de problemas en hombres y animales (para una revisión crítica, véase Carretero y García Madruga, 1984; Mayer, 1983). Esta tradición ha rebrotado durante la década de los setenta, tanto en el razonamiento lógico (Evans, 1978; 1984; Wason, 1983) como en el estadístico (Tversky y Kahneman, 1974), además de extenderse a áreas como la solución de problemas - centrada ahora en la comparación entre expertos y novatos (Chi, Glase y Rees, 1982; Simon y Simon, 1978; para una revisión véase Pozo, 1987) - o la psicología social (Nisbett y Ross, 1980). En un sentido más general estos modelos se desarrollan al tiempo que la psicología cognitiva cambia parcialmente su rumbo con los nuevos aires traídos por los teóricos del esquema, para quienes comprender un problema y resolverlo es prácticamente lo mismo (Rumelhart, 1980).

Con objeto de poner a prueba estos tipos de modelos se han realizado múltiples investigaciones sobre la ainfluencia del contenido en el razonamiento». En términos generales, y de acuerdo con los supuestos del dualismo, estas investigaciones han partido de la idea de que la forma y el contenido del razonamiento son variables independientes entre sí y que, por tanto, en un marco experimental pueden ser manipuladas convenientemente con el fin de determinar su importancia relativa. Pero, partiendo de esta idea común, existen serias diferencias dentro de los estudios sobre «la influencia del contenido en el razonamiento», que no siempre son consideradas, llevando a generalizaciones abusivas. De hecho, cada uno de los tipos de modelo que hemos reseñado suele apoyarse en una literatura propia, por lo que, en realidad, pueden no ser incompatibles y responder a la solución de distinto tipo de problemas. Unos trabajos estudian la diferencia entre tareas con y sin contenido temático; otros se ocupan de las diferencias entre diversos tipos de contenidos temáticos (sobre todo causales y no causales); otros estudian el razonamiento en diferentes dominios de conocimiento, científicos y «naturales», así como la familiaridad de los sujetos con esos diversos dominios y, finalmente, otros se ocupan de las relaciones entre las expectativas del sujeto y su razonamiento. Pero también hay datos en sentido inverso: influencia del razonamiento lógico sobre las ideas previas de los sujetos, si bien son muy escasos.

Al revisar, de un modo muy somero, cada uno de estos trabajos intentaremos mostrar la falsedad del supuesto básico del que parten: la independencia de forma y contenido en el razonamiento, proponiendo en cambio un modelo interactivo, que supone de hecho una tercera vía que pretende integrar las dos posiciones anteriores, concibiendo el pensamiento como una interacción entre dos tipos de información, que no se excluyen sino que al contrario se exigen mutuamente: la información disponible en el medio, de la que se pueden inferir conclusiones mediante reglas de razonamiento, y la información acumulada en la memoria. Lá importancia de una y otra fuente de información estará regida con criterios funcionales o pragmáticos. Esta interacción de forma y contenido plantea, en nuestra opinión, un serio reto a la psicología del procesamiento de información, incapaz de afrontar de forma satisfactoria la existencia de contenidos no reducibles a reglas formales (por ej., Gardner, 1985). 


\section{INFLUENCIA DEL 'CONTENIDO’ DE LA TAREA SOBRE EL RAZONAMIENTO}

\section{Tareas con y sin contenido temático}

Son muchas las investigaciones que se han ocupado de estudiar la influencia del contenido temático sobre el uso de reglas formales de razonamiento lógico. Generalmente suelen comparar el rendimiento de un mismó sujeto (o a veces de sujetos diversos con características similares) en dos versiones de una misma tarea - por tanto con la misma estructura lógica一, una con contenido temático y la otra sin contenido (abstracta).

De entre las diversas tareas utilizadas en este tipo de investigaciones sin duda la más documentada, hasta el punto de constituir ya un área de investigación en sí misma, es la famosa tarea de selección (o tarea de las cuatro tarjetas) ideada por Wason hace ya veinte años. En la versión original de esta tarea (véase, por ej., Wason y Johnson-Laird, 1972), el sujeto tenía delante cuatro tarjetas que, por su cara visible, mostraban respectivamente una $\mathrm{E}$, una $\mathrm{K}$, un $4 \mathrm{y}$ un 7 (véase figura 1). Al sujeto se le informa que cada tarjeta contiene por una cara una letra y por la otra un número. A continuación se presenta una regla referida a esas cuatro tarjetas según la cual «si una tarjeta tiene una vocal en una cara, entonces tiene un número par en la otra cara». Lo que el sujeto tiene que hacer es seleccionar aquellas tarjetas y sólo aquellas tarjetas que es necesario dar la vuelta para comprobar si la regla es verdadera o falsa. La solución correcta es E y 7 (o, en notación lógica, $p$ y no-q). Sin embargo sólo entre un 10 y un $20 \%$ de los sujetos, incluso estando entrenados en lógica, suelen dar esta respuesta. La elección más frecuente es $\mathrm{E}$ y 4 , que conlleva dos errores, uno de comisión (seleccionar $q$ ) y otro de omisión (no elegir $n \circ-q$ ). Inicialmente esta respuesta fue interpretada como una tendencia ilógica a la verificación del enunciado en lugar de a su falsación. Posteriormente ha sido reinterpretada en términos no-lógicos, como una tendencia al emparejamiento de los dos casos explícitamente citados en el enunciado del problema (Evans, 1972; Wason y Evans, 1975).

FIGURA 1
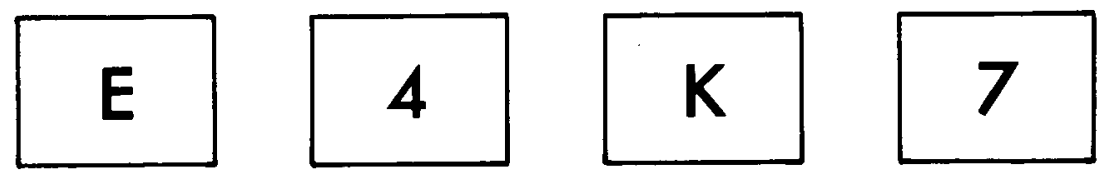

El sujeto debe comprobar el enunciado condicional «su una tarjeta tiene una vocal en una cara, entonces tiene un número par en la otras. 
Pero junto a esta sorprendente dificultad de la tarea se observó pronto que se veía considerablemente facilitada con la sola introducción de un contenido temático en las tarjetas. Cuando Johnson-Laird, Legrenzi y Sonino-Legrenzi (1972) sustituyeton las letras y los números por cartas con mayor o menor franqueo según su procedencia, comprobaron que el $81 \%$ de los sujetós hacían la elección correcta. De esta forma, durante toda la década de los setenta se sostuvo que el contenido tenía efectos facilitadores en sí mismo, ayudando al sujeto a razonar mejor que cuando la tarea era abstracta. Se llegó a postular incluso que este efecto refutaba por sí mismo la teoría piagetiana del pensamiento formal, ya que los sujetos no razonaban sobre la forma sino a partir de contenidos concretos (Wason, 1977).

Sin embargo, cuando la influencia del contenido en la tarea de selección se retomó a finales de la década pasada, el efecto hasta entonces observado se diluyó. Se intentaron replicar aquellos primeros trabajos sin éxito, comprobándose que no siempre el contenido familiar facilita la tarea (para una revisión de estos trabajos más recientes véase Evans, 1982; Griggs, 1983; Wason, 1983). Según estos trabajos, el efecto del contenido familiar no es propiamente facilitar el uso de reglas de razonamiento, sino ayudar a la evocación de un contraejemplo tomado de la memoria a largo plazo, que permite solucionar el problema falsando la regla. Por tanto la tarea de selección no es ya una tarea de razonamiento sino de memoria. La evocación estaría ligada al contenido específico del problema y al «espacio del problema que el sujeto activa como consecuencia de las instrucciones recibidas al comienzo. Actualmente, se considera que los factores relevantes para la solución del problema de las cuatro tarjetas son los indicios de memoria y las instrucciones proporcionadas al sujeto (Chrostowski y Griggs, 1985; Griggs, 1984; Valentine, 1985; Yachanin, 1986). En otras palabras, la tarea de selección no se considera ya una tarea de razonamiento, sino de evocación de contraejemplos. El sujeto debe recuperar conocimientos de su memoria. Cuando los conocimientos evocados por el contenido no fueron falsadores sino verificadores, el contenido en vez de ayudar entorpecería.

Pero la reducción del razonamiento a un problema de memoria plantea muchos problemas. En primer lugar, hay ocasiones en que el sujeto no logra falsar una hipótesis aún cuando tenga un contraejemplo presente, especialmente cuando está en juego ideas mantenidas firmemente por el propio sujeto, como veremos más adelante en este mismo artículo. En segundo lugar, no todas las respuestas correctas son explicables por evocación de conocimientos específicos (Cheng y Holyoak, 1985). Y, por último, la memoria no está tan alejada de los conocimientos lógicos como supone esta interpretación. Si aceptamos su naturaleza constructiva estamos vinculándola en mayor o menor medida a los procesos de razonamiento lógico (Falmagne, 1975). En último caso, ¿cómo sabe el sujeto que ese hecho es un contraejemplo? La contradicción es sin duda una categoría lógica y no fáctica (Millar, 1982).

Esta interacción entre representación y razonamiento en la tarea de selección se ve reforzada por algunas interpretaciones recientes de la misma. Básicamente, se empieza a postular que la representación evocada por el contenido específico no es, sin embargo, una representación específica, sino más bien una representación «unitaria» de la tarea (Wason y Green, 1984). Ello equivale a reconocer que la introducción del contenido cambia por completo el problema, de forma que ya no es la misma tarea. En otras palabras, supone admitir que la estructura de la tarea para el sujeto («el espacio del problemas) cambia cuando 
cambia su contenido. Esto es, contendio y estructura son dos aspectos inseparables y solidarios. En esta misma línea, Cheng y Holyoak (1985) han propuesto recientemente que lo hace el sujeto es activar un esquema pragmático de naturaleza general y no específica (por ej., «permiso», «obligación» o «causación»), cosa que no es posible en la tarea sin contenido temático, dado que esa tarea, más que abstracta es arbitraria y por tanto no permite la aplicación de esquemas pragmáticos. Por ello la solución de tareas arbitrarias y significativas se basa en procesos diferentes y puede afirmarse que, desde un punto de vista psicológico, son tareas diferentes. Cheng y Holyoak (1985) señalan también algunas diferencias entre los diversos significados pragmáticos que puede adquirir la tarea. Uno de ellos es la causalidad. ¿Qué influencia tiene la introducción de nexos causales en el contenido de una tarea?.

\section{Tareas causales y no causales}

De entre los contenidos temáticos que suelen incluirse en la tareas de razonamiento, una buena parte de ellos suelen implicar relaciones causales. Los conocimientos causales son además muy relevantes en la vida cotidiana de las personas, ya que éstas no sólo tratan de describir los hechos sino también explicarlos y comprenderlos. Esas explicaciones se alcanzan muchas veces, aunque no siempre, por la vía de la causalidad. Por ello puede afirmarse que buena parte de nuestro pensamiento tiene un contenido causal, por lo que resulta interesante aclarar la influencia de este tipo de contenidos sobre el razonamiento (sobre las relaciones de la causalidad con el razonamiento y sobre la propia naturaleza de la causalidad véase Pozo, 1986).

Las investigaciones incluidas en este apartado suelen comparar el rendimiento del mismo sujeto (o de sujetos equivalentes) en una misma tarea (o dos tareas semejantes) con dos contenidos temáticos distintos: uno claramente causal y otro no causal. Normalmente esos contenidos son muy próximos entre sí.

Cheng y Holyoak (1985) sugieren que el uso de un esquema pragmático causal en la tarea de selección obstaculiza su solución correcta, al incrementar la falacia de afirmación del consecuente, ya que, según ellos, las relaciones causales suelen entenderse de un modo biunívoco. Sin embargo, Moshman (1980) ha obtenido resultados que no se ajustan del todo a esta hipótesis, ya que la introducción de un contenido causal facilita la tarea, si bien entre los adultos sigue habiendo interpretaciones reversibles del condicional. Lawson (1983a) corrobora plenamente la hipótesis al comprobar que la introducción de un contendio causal incrementa las interpretaciones bicondicionales. En cualquier caso, esta interpretación bicondicional de los enunciados causales puede verse notablemente favorecida por la propia disposición de la tarea, que no proporciona ninguna causa potencial alternativa, y no por el nexo causal en sí mismo (véase Pozo, 1987). De hecho, a pesar de su interés, la tarea de selección parece tener muy escasa relación con otras tareas causales más próximas a la vida real (Lawson, 1983b). Conviene por tanto analizar la influencia de la causalidad en ese otro tipo de tareas.

Tversky y Kaheman (1982) han constatado que la información causal tiene un mayor impacto sobre el sujeto que la información no causal. Así, mientras la información cuantitativa es habitualmente despreciada para hacer una predicción cuando se dispone de información cualitativa, sin embargo cuando esa 


\section{4}

información cuantitativa es causal -es decir está conectada causalmente con el hecho que hay que predecir- los sujetos la tienen en cuenta. Este mayor impacto de la información causal podría explicar sus efectos sobre el aprendizaje y la memoria. En términos generales resulta más facil de aprender una relación entre dos hechos cuando es causal (Ausubel y Shiff, 1954; Bindra, Clarke y Shultz, 1980; Copple y Coon, 1977). Del mismo modo, la cohesión causal desempeña una función muy importante en el aprendizaje de textos escritos (Trabasso, Secco y Van Den Broeck, 1984) y como recurso mnemotécnico en la asociación de palabras (Lindsay y Norman, 1977).

Ahora bien, esta facilidad para el recuerdo de las relaciones causales, que podría interpretarse como una prueba de que la causalidad supone una reelaboración de los datos que implica un nivel más profundo de procesamiento, no se traduce per se en una facilitación de razonamiento, sino al contrario. Crocker (1981) sugiere que el uso correcto de la estrategia de correlación es aún más improbable cuando ésta se entiende de un modo causal. Adi et al. (1978) proporcionan datos favorables a esta sugerencia, al mostrar un peor uso de la correlación en tareas causales que en tareas con relaciones arbitrarias entre las variables. Según los autores, esta diferencia sería debida a que en la tarea causal el sujeto va guiado por una hipótesis que trata de comprobar, lo que le hace centrarse más en unos datos olvidándose del resto que, formalmente, tienen la misma relavancia. En la misma línea, Mattehws y Sanders (1984) comprobaron que cuando los datos responden a relaciones causales los sujetos utilizan criterios temporales y los remiten a sus conocimientos previos en mayor medida que si no son causales. Los autores concluyen que dos tareas equivalentes en su forma, una causal y otra no causal, son en realidad cualitativamente distintas para el sujeto.

En resumen, no puede afirmarse que la presencia de un contendido causal haga más fácil el uso de las reglas de inferencia causal. El fuerte significado que para los sujetos tienen los datos causales induce búsquedas menos exhaustivas, guiadas por expectativas. Esas expectativas darán lugar a un uso adecuado de las estrategias cuando se reunan dos condiciones: a) que estén en la misma dirección de los datos presentados; y b) que la tarea no requiera un análisis completo de todos los datos presentes, sino más bien la aplicación de un herístico selectivo previo. En cualquier caso, la introducción de un contendido causal en una tarea parece modificar sustancialmente la estructura de la tarea a la que el sujeto cree enfrentarse. Una vez más, forma y contenido interactúan en el razonamiento.

\section{Tareas de diversos dominio de conocimiento}

Otro tipo de estudios con respecto a la influencia del contenido sobre el razonamiento consisten en la presentación a un mismo sujeto (o sujetos equivalentes) de dos tareas con estrutura lógica similar (o parecida) pertenecientes a dos dominios distintos. Hay dos tipos de comparaciones relevantes: las de tareas «naturales» (esto es, cotidianas) con tareas científicas (de ciencia físicas o naturales) y las que comparan entre sí tareas de contenido científico físico y social.

\section{Tareas cientificas fisicas y sociales}

Si bien la mayor parte de las tareas de razonamiento con contenido científico recurren a las ciencias físicas o naturales, debido sin duda a que su naturaleza 
más precisa facilita el análisis de las tareas, últimamente se está despertando también el interés por el razonamiento en problemas sociales. Así se han estudiado, ya sea en niños o en adultos, el conocimiento pisológico (por ej., Dweck y Bempechat, 1983; Whiteman, 1967, 1970; o también dentro de la teoría de la atribución, véase Harvey y Weary, 1984; Kelley y Michella, 1980), moral (por ej., Piaget, 1932) económico y político (por ej., Adelson, 1971; Delval, 1981) e histórico. En este último área, se han identificado un conjunto de ideas o contenidos relacionados con la causalidad, como el tiempo histórico (Carretero, Pozo y Asensio, 1983; Pozo, 1985a), los conceptos sociohistóricos (Adelson, 1971; Carretero, Pozo y Asensio, 1983; Pozo y Carretero, 1984) o la relatividad de las explicaciones históricas (Asensio, Carretero y Pozo, 1986; Kuhn, Pennington y Leadbeater, 1983).

Pero todos los trabajos que hemos venido reseñando se ocupan más bien de las ideas que poseen los sujetos en diversos ámbitos del conocimiento social. Ahora bien ison las reglas de razonamiento causal las mismas en los diversos dominios de conocimiento? No existen muchos datos al respecto. Por ejemplo, los que hay disponibles con respecto al razonamiento en contextos históricos muestran que tiene indudables rasgos comunes con el razonamiento en situaciones físicas según el modelo piagetiano de las operaciones formales (Jurd, 1978; Pozo y Carretero, 1983). Sin embargo, estos trabajos se limitan a aplicar un solo tipo de tarea a los sujetos, sin que haya propiamente comparación entre tareas de diversos dominios. Cuando esas comparaciones se han efectuado, han surgido algunas diferencias de diversa consideración. La mayor parte de estos trabajos se han centrado en comparaciones de tipo cuantitativo que ofrecen escasa información. En un trabajo de mayor interés, Lou (1979) comparó el rendimiento de sujetos adolescentes y adultos en tareas de control de variables con conteni-

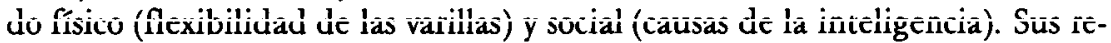
sultados mostraban que los sujetos usaban el mismo tipo de razonamiento en ambos dominios, con una alta consistencia interna. Igualmente, no existían diferencias globales entre ambos dominios, que mostraban pautas evolutivas semejantes. Sin embargo, sí aparecieron ciertas diferencias de tipo cualitativo entre ambos dominios, que Lou (1979, pág. 128) resume del siguiente modo:

a) Los sujetos tienen más dificultades para comprobar la relevancia de las variables en la prueba social.

b) Las variables sociales resultan más difíciles de definir y por tanto de controlar.

c) Las relaciones entre variables en la prueba social son más complejas (interacciones e interconexiones) por lo que no se pueden cuantificar.

Resulta sorprendente, a simple vista, que estas diferencias cualitativas, sin duda importantes, no se traduzcan en diferencias cuantitativas. Ello se debe tal vez a que, al igual que en otros de los trabajos reseñados (por ej., Jurd, 1978), la autora adoptó un enfoque piagetiano que, como es sabido, tiende a minimizar las diferencias cualitativas en la interpretación de los resultados en favor de una caracterización más global de los mismos. De hecho, cuando se han introducido análisis más minuciosos, separando los aspectos inferenciales o formales de los contenidos o conceptos, se han observado diferencias cuantitativas claras entre uno y otro tipo de tareas científicas (Pozo, 1985b). Así, en las tareas históricas resulta notablemente más difícil aplicar estrategias de razonamiento, que 
en cambio son fácilmente aplicadas a tareas físicas. Ello se debe a que en las tareas históricas la diferenciación de las variables intervinientes en un hecho es siempre de naturaleza conceptual y nunca puede basarse en indicios perceptivos facilitadores como en las tareas físicas. Sin entrar a analizar con mayor detalle estos y otros datos de la misma investigación (Pozo, 1985b) la mayor dificultad en el razonamiento parece deberse a la propia naturaleza de los contenidos históricos y sociales. Una vez más no hay razonamiento independiente del contenido, pero tampoco éste es un efecto nocivo «a posteriori», sino que forma parte de la propia estructura de la situación.

La existencia de esas diferencias en la naturaleza de la causalidad social hace imposible el propósito inicial en algunos de estos trabajos de comparar tareas de «estructura isomorfas y contenido diverso (físico o social). Nuevamente podemos comprobar que la forma y el contenido de un razonamiento están estrechamente conectados requiriendo un análisis interactivo de los procesos de representación y razonamiento.

\section{Tareas naturales frente a tareas cinetíficas}

El mismo propósito - comparar tareas isomorfas de contenidos diversoshan guiado también las investigaciones incluidas en este apartado. En este caso se ha tratado de comparar una tarea científica de control de variables - generalmente una tarea de contenido físico tomada de Inhelger y Piaget (1985)con una tarea de estructura lógica idéntica, cuyo contenido era supuestamente cotidiano o no científico (por ej., el cuidado de unas plantas, las ventajas de una pasta de dientes o la forma más efectiva de pescar). No obstante, las tareas científicas permitían el control de variables mientras que habitualmente las tareas cotidianas requerían más bien evaluar covariaciones observadas, aspecto al que se ha prestado menos atención de la que se merece.

Dos grupos de investigación han abordado estos trabajos. Por un lado, Kuhn y colaboradores han comparado la tarea de las plantas, consistente en determinar cuáles de los factores presentados (agua, aceite para las hojas, vitaminas y abono) determinan el estado de salud de cuatro plantas (véase Kuhn, Pennington y Leadbeater, 1983), con la tarea piagetiana del péndulo, en la que el sujeto debe determinar también cuáles de los factores presentes (peso, longitud de la cuerda, amplitur y fuerza de los impulsos) determina la velocidad de oscilación de un péndulo. Los resultados obtenidos variaron de un trabajo a otro. Mientras en una ocasión resultó más fácil la tarea del péndulo (Kuhn y Brannock, 1977) en otra sucedió lo contrario, siendo más frecuente el control de variables en la prueba de las plantas (Kuhn, Capon y Carretero, 1983). Estas diferencias pueden atribuirse al diferente tipo de sujetos empleados en cada caso: mientras en la primera investigación citadas eran adolescentes, con una cierta familiaridad en la resolución de tareas científicas escolares, en la segunda eran amas de casa con un nivel de estudios bajo o medio, por lo que cabe pensar que estarían más acostumbradas a la solución de problemas domésticos. Esta interpretación se ve también apoyada por el grupo de investigaciones dirigido por Linn, de Benedictis y Delucchi 1982) y otros problemas de laboratorio (por ej., Linn, Clement y Pulos, 1983). Estas diferencias cuantitativas resultan no obstante insuficientes, ya que no permiten acercarse cualitativamente a las diferencias entre ambos dominios de conocimiento, tal como sucedía en el caso del conocimiento social. Aún así, en estos trabajos, a diferencia de los anteriores, había 
un control previo de las expectativas del sujeto con respecto a las diversas variables presentes, lo que permite establecer una relación directa entre él número de variables controladas y el número de variables que el sujeto cree relevantes. Según los autores esas creencias varían de una tarea a otra en función de la familiaridad que los sujetos tienen con ellas, prediciendo una influencia facilitadora de la familiaridad que, sin embargo, debe aún ser demostrada.

\section{Influencia de la familiaridad con la tarea}

El supuesto de que la familiaridad o la cantidad de práctica con una tarea facilitan su resolución es compartido por muchos modelos en psicología del pensamiento. Así, por ejemplo, además de ser recogido en la tradición conductista, la psicología del procesamiento de información asume que, como efecto de la práctica, el sujeto elabora chunks o trozos de información que dejan libre una parte de su capacidad de procesamiento para otros procesos. Esta idea subyace de alguna forma a los estudios sobre expertos y novatos (Chi, Rees y Glaser, 1982; también Pozo, 1987) y ha sido retomada también por los autores neopiagetianos en psicología del desarrollo (Case, 1985). El propio Piaget (1970b) se adhirió a la hipótesis de la familiaridad para explicar la no universalidad del pensamiento formal. Sin embargo, los efectos de la familiaridad con una tarea resultan, aún hoy, poco claros.

Las investigaciones que han intentado elucidarlos han consistido en la presentación a dos grupos de sujetos de dos tareas de estructura semejante y contenido diverso, con respecto al cual los dos grupos tenían una familiaridad distinta. Así, Pulosy Linn (1981) presentaron a dos grupos de adolescentes, unos urLañús y ơtios de uña zona rúal rica en pesca, varias tareas científicas de contro! de variables (varillas, péndulo, rampa, etc.), dos pruebas de contenido neutro y una tarea de evaluación de la covariación relacionada con la pesca. Conforme a las previsiones, los sujetos rurales rindieron más en la tarea de pesca mientras que los urbanos lo hicieron globalmente mejor en las tareas científicas, si bien en algunas tareas científicas no hubo diferencias. Las tareas neutras no mostraron ninguna diferencia.

Pero los resultados de este tipo de experimentos no son tan claros como parece. En primer lugar, como señala Carretero (1980) ¿de qué forma se determina la familiaridad previa de los sujetos? En el caso citado se ha resultado este problema recurriendo a un enfoque ecológico. Pero si este enfoque puede ser válido para determinar la familiaridad con respecto a la tarea de la pesca, no queda claro en qué medida es útil en el caso de las tareas científicas. ¿A qué se deben las diferencias entre unas tareas científicas y otras? Por ejemplo, mientras en la rampa ambos grupos diferían claramente, en el péndulo esas diferencias eran mínimas. ¿Se debe a que los sujetos urbanos no tenían la misma familiaridad con ambas tareas? Es esta una explicación plausible, pero a a posteriori, ya que la familiaridad no fue medida previamente.

De hecho, la supuesta ventaja de la familiaridad está poco fundamentada en datos empíricos. Así, hay pruebas de que sujetos con escasa o nula formación académica se muestran incapaces de aplicar un razonamiento formal esto es al margen del contenido- tanto en tareas muy familiares como es el cuidado de las plantas (por ej., Kuhn, Capon y Carretero, 1983) como en tareas con las que no tienen ninguna familiaridad, como mostró el famoso trabajo 


\section{8}

de Luria (1974) en el Uzbekistán. Este tipo de datos parecen apoyar la relación curvilínea entre familiaridad y razonamiento propuesta por Linn (1980; véase Carretero, 1980). Según esta relación el razonamiento sería más eficaz en niveles medios de familiaridad, ya que en las tareas muy familiares se prodüce una interferencia de las ideas sobre los procesos inferenciales. Sin embargo, tampoco esta hipótesis se ve apoyada por los datos. El rendimiento máximo de los sujetos rurales en el trabajo de Pulos y Linn (1981) se producía en la tarea de la pesca, que era supuestamente la más familiar para ellos. ¿Qué sucede entonces? ¿Cuál es la influencia de la familiaridad? En nuestra opinión, la familiaridad o la práctica son variables que - en el caso de llegar a medirse - no son en sí mismas determinantes del pensamiento del sujeto. Esto no mantiene ninguna relación universal y constante con la práctica. Por ejemplo, en algunas áreas de conocimiento, como por ejemplo las ideas sobre el movimiento de los objeos (Pozo, 1987), se ha observado que uno de los factores que entorpecen la evolución de los conocimientos de los sujetos, interfiriendo en su razonamiento, es precisamente la práctica continuada que los sujetos tienen con problemas de movimiento. Este efecto «nocivo» de la práctica, reminiscente del concepto de fijeza funcional de los autores dela Gestalt, no se produce sin embargo con todos los contenidos. En definitiva el problema no es la cantidad de práctica que tenga un sujeto con una tarea sino la naturaleza de esa práctica. En otras palabras, lo relevante son las ideas generadas por el sujeto con respecto a la tarea como consecuencia de esa práctica o familiaridad.

\section{Influencia de las ideas previas}

La influencia de las ideas previas se ha estudiado de dos formas distintas. En una de ellas se presentan a un mismo sujeto dos tareas análogas con respecto a las cuales posee ideas de distinta calidad. En la otra se presenta una misma tarea a sujetos con distintos conocimientos o ideas previas al respecto. En cualquiera de los casos, idealmente parece necesario un control previo de las ideas de cada sujeto con respecto a las tareas propuestas, lo cual plantea ciertos problemas metodológicos.

La influencia de las expectativas del sujeto sobre el razonamiento es hoy un lugar común en psicología del pensamiento. Practicamente en todas las tareas y en todas las áreas ha sido comprobada esta influencia. Se ha comprobado que esa influencia de las ideas previas afecta a todas las fases o estadios del razonamiento o solución de un problema, desde su formulación a la búsqueda de datos relevantes y a la interpretación de los mismos. La propia percepción de los hechos sobre los que se han de aplicar las reglas está mediatizada por las expectativas. Esto es especialmente notorio en el caso de que esos hechos sean de naturaleza social, ya que suelen ser más ambiguos y susceptibles de interpretación. Por ejemplo, un fenómeno en el que se ha estudiado abundantemente esta percepción sesgada es en la eformación de impresiones» o atribución de rasgos conductales y cognitivos a los otros. Parece haber un considerable efecto de primacía por el que la primera impresión provocada por una persona tiene una fuerza mucho mayor que la información posteriormente recogida, aun cuando ésta sea patentemente contradictoria. Esa primera impresión produce una codificación selectiva de toda la información que posteriormente se recoge sobre esa persona (Ross, 1977). Sucede así incluso cuando la base informativa so- 
bre la que se ha formado esa primera impresión es muy estrecha. Se ha comprobado que una sola información basta para formar expectativas sólidas (Read, 1983) y que esas expectativas perduran incluso cuando esa estrecha información sobre la que se basaban sea claramente desacreditada por no ser significativa o representativa (Ross, 1977). Es como si las expectativas formadas fueran autónomas con respecto a los datos que supuestamente las apoyan. Esta observación de Ross (1977) muestra una vez más la insuficiencia de los análisis «lógicos» de regularidades en el estudio del razonamiento. Las expectativas pueden persistir incluso sin datos objetivos que las apoyen. Esta persistencia se consolida además mediante los procesos de búsqueda selectiva de nueva información con respecto a las expectativas. Snyder (1981) ha mostrado la existencia de un sesgo verificador en la búsqueda de una nueva información con respecto a una expectativa, que hace difícil el encuentro con información falsadora. Pero incluso cuando el sujeto «tropieza» con información contraria a sus expectativas suele reinterpretarla de forma que resulte congruente con las mismas. Así, cuando una personas actúa de forma muy distinta a los que esperamos de ella, atribuimos esa conducta anómala a factores situacionales externos y no a rasgos disposicionales internos (Kulik, 1983), en una inversión del llamado serror fundamental de atribución» (Ross, 1977), consistente en la tendencia a hacer atribuciones internas donde corresponden atribuciones externas. A estos sesgos cognitivos se une además la carga afectiva que suele acompañar a las atribuciones sociales, que pueden disminuir aún más la fuerza de los procesos inferenciales, ya que, como afirma Zajonc (1980) "las preferencias no necesitan inferencias».

Pero estas tendencias no son exclusivas de los procesos de atribución psicológica. Se producen también cuando se trata de atribuir casualidad a objetos o hechos físicos. También aquí existe una codificación selectiva en función de las ideas previas por la que dos sujetos no ven lo mismo enfrentados a un mismo hecho. Así, por ejemplo, ante un simple problema de movimiento un experto y un novato en física no ven lo mismo (Pozo, 1987); en un caso observan fenómenos físicos (por ej., la conservación de la cantidad de movimiento) y en otro objetos y sucesos reales (por ej., dos bolas que chocan). Igualmente, la búsqueda de información que ponga a prueba las propias ideas es selectiva, favoreciendo procesos de verificación. Linn (1983; Linn, Clement y Pulos, 1983) ha mostrado que, en una situación de control de variables, los sujetos sólo someten a prueba aquellas variables que, según sus expectativas, influyen, no controlando por el contrario el resto de las variables presentes. Este sesgo verificador en la búsqueda de información suele acompañarse además de resistencias a la falsación cuando se encuentran con información contraria a las expectativas. En una investigación sobre las causas de la flotación de los cuerpos Carretero (1979, 1984) presentó a sujetos de 7 a 17 años unos recipientes con agua y unas bolas de diversos materiales y tamaños, acompañadas de unas tarjetas que formulaban hipótesis sobre las causas de la flotación. Al sujeto se le pedía que comprobase con el material si esas hipótesis eran verdaderas o falsas. Previamente con un grupo equivalente de sujetos se había establecido la secuencia evolutiva de explicaciones causales con respecto a esa noción, comprobando que el peso ocupaba un lugar fundamental en las explicaciones, especialmente entre los niños de edades intermedias. De esta forma se pudo comprobar que las tendencias verificacionistas eran mayores en el caso de que la hipótesis expresase una idea plausible para el sujeto. Cuando esta idea era contradicha por algún dato muchas veces no se rechazaba la hipótesis sino que se consideraba la observación 
como un caso particular por el que la hipótesis era «verdadera y falsa a la vez».

Este mismo tipo de resultados han sido observados, aparte de en el ya clásico trabajo de Karmiloff-Smith e Inhelder, (1975), en otras investigaciones sobre nociones distintas, por ejemplo históricas (Pozo y Carretero, 1983). En un sentido más general la tendencia a la verificación de las propias ideas parece estar presente en la propia labor científica de los psicólogos. Si hacemos caso a Greenwald (1975) hay en la investigación psicológica un auténtico «prejuicio contra la hipótesis nular, por la que esta, cuando se formula, suele ser teóricamente irrelevante.

Tomados en conjunto todos los datos anteriores, podría decirse que, en la mente del sujeto, hay una auténtica conspiración contra el razonamiento, que éste es inoperante y que por tanto el pensamiento podría reducirse a la recuperación de esquemas o ideas de la memoria. Pero ésta es sólo una parte del cuadro. Lo cierto es que la mayor parte de la investigación sobre la influencia de las ideas previas en el razonamiento se ha hecho en el seno del debate sobe la racionalidad lógica del ser humano y ha tendido a enfatizar aquellas conductas que se desviaban de los modelos normativos. Pero posiblemente la realidad se halle lejos de esos cuadros un tanto catastrofistas (por ej., Nisbett y Ross, 1980). La conspiración no ha triunfado. El predominio de las teorías o ideas previas de los sujetos con respecto a sus reglas de razonamiento (lógico o ilógico) no conlleva una supresión efectiva de éstas últimas. Los sujetos razonan, peto desde ideas o teorías fuertemente arraigadas. Probablemente, el pensamiento esté regido por criterios pragmáticos y no por criterios lógicos. Analizados desde ese punto de vista muchos de los sesgos observados son en realidad la conducta más sensata en esa situación. Por ejemplo, la atribución a factores situacinales de aquellas conductas que se desvían de nuestras previsiones nos permite seguir teniendo modelos de esas personas que nos ayuden a controlar nuestras interacciones con ellas. Del mismo modo el abandono de una teoría sobre el movimiento o la flotación de los objetos ante la aparición de un primer contraejemplo resulta poco eficaz a no ser que tengamos una teoría mejor. Aunque falle en ocasiones, la teoría de que los objetos flotan porque pesan poco es de una utilidad bastante general, sobre todo si tenemos en cuenta la percepción propioceptiva del peso. Si poseer una teoría fuerte sesga nuestra percepción del mundo, al destacar unos objetos y relaciones en detrimento de otros, la ausencia de teorías no parece producir un precesamiento mejor. De hecho cuando no se tiene una buena teoría, el recuerdo (Eylon y Reif, 1984) y el razonamiento (Wright y Murphy, 1984) son peores que cuando se tienen. En otras palabras, más vale una mala teoría, aunque se base en observaciones ilusorias (Chapman y Chapman, 1967, 1969), que la falta de teoría. Y este suele ser el dilema que en muchas investigaciones psicológicas se presenta a los sujetos.

Según esta formulación, el problema no reside en las expectativas aisladas de los sujetos, en las que se han basado todos los estudios anteriores, sino más bien en la organización de conjunto de la que forman parte esas expectativas, a la que en este trabajo venimos denominando teorías. Buena parte de la resistencia a falsar las propias ideas provienen de que esta falsación no consiste en un simple cambio en el valor de verdad de un enunciado, sino en una reorganización de todo un sistema de ideas. El estudio de las teorías causales en situaciones de razonamiento puede ayudar a entender mejor la naturaleza de ese razonamiento. 
Pero ¿qué relación existe entre el razonamiento, entendido como el análisis «lógico de los hechos observados, y las ideas de los sujetos con respecto a esos mismo hechos? ¿Logra el razonamiento modificar esas ideas? Y si no es así, ¿de dónde proceden dichas ideas? Para responder a estas preguntas hay que estudiar no sólo la influencia del contenido sobre el razonamiento sino también, a la inversa, la fuerza que el razonamiento tiene para cambiar las teorías de los sujetos.

\section{INFLUENCIA DEL RAZONAMIENTO SOBRE LAS IDEAS PREVIAS}

En un trabajo sobre el uso de la detección de la correlación, Jennings, Amabile y Ross (1982) hacían dos observaciones que, tomadas conjuntamente, resultan sorprendentes. Por un lado observaban que los sujetos eran bastante imprecisos en sus estimaciones correlacionales cuando se les presentaban unos datos. Por otro comprobaban que sus ideas, en ausencia de datos, con respecto a la correlación entre diversas variables conocidas se ajustaban notablemente a la realidad. ¿Dónde sugían estas teorías precisas? ¿Cómo pueden compaginarse con la impresión de las estimaciones?

Surge una respuesta posible: el uso de las reglas de razonamiento no tiene relación con la génesis de las ideas o teorías. Según esta idea, el pensamiento, como las preferencias (Zajonc, 1980), tampoco necesita inferencias. Es sólo memoria. Pero esta interpretación extrema no se ajusta a los datos que conocemos. Los sujetos son sensibles a la información del medio y realizan inferencias a partir de ella. El problema es que estas inferencias no sólo se basan en la «forma» lógica de esa información, sino también en su contenido declarativo.

En el otro extremo, esas teorías pueden atribuirse precisamente a la acción de esas reglas de inferencia. Es la clásica posición empirista, según la cual el origen de las teorías reside en la detección de covariaciones regulares (Alloy y Tabachnik, 1984; Crocker, 1981; Kelley, 1973). Cuando esas covariaciones dejan de producirse la teoría se extingue (Alloy y Tabachnik, 1984). Pero tampoco esta posición parece sostenible. La evolución de las teorías causales posee caracteres propios que hacen difícil el cambio inductivo o «por descubrimiento» (Pozo, 1987). De hecho, según Lakatos (1978) ni siquiera en la ciencia, donde los recursos procedurales para la aplicación de reglas de razonamiento son más refinados, se produce de modo habitual el cambio inductivo, puesto que las teorías no son sustituidas cuando tropiezan con datos en contra sino cuando aparece otra teoría mejor. No obstante, si bien los datos no inducen cambios inmediatos en las teorías, tienen una influencia más lenta, de carácter acumulativo, por la que, por decirlo de alguna forma, van minando las resistencias de la teoría al cambio, conjugandose así la naturaleza a la vez cuantitativa y cualitativa del cambio conceptual (véase al respecto Pozo, 1987).

En definitíva, aunque sin duda el razonamiento no es una condición suficiente para el progreso teórico, es posible que sea una condición necesaria. Algunos estudios microgenéticos (Karmiloff-Smith e Inhelder, 1975; Kuhn y Phelps, 1982) indican que el razonamiento reiterado con un material acaba por producir cambios conceptuales, si bien estos son más lentos de lo que un modelo racional predeciría y no siempre se producen. 


\section{2}

\section{A MODO DE CONCLUSION}

El conjunto de datos que hemos revisado nos permiten afirmar que contrariamente a los supuestos logicistas del paradigma cognitivo imperante del procesamiento de información, el pensamiento no puede reducirse exclusivamente a su forma o «sintaxis». Es necesario considerar también el contenido de las tareas, que no parece reducible a reglas formales estandarizadas. Esta introducción del contenido supone en nuestra opinión un serio reto para el paradigma cognitivo, al tiempo que un cambio esperanzador. Con el contenido entran en la psicología cognitiva muchos de los fantasmas que estaban expulsados por ella. Hasta ahora, se ha supuesto que el razonamiento se apoyaba en una lógica binaria o bivalente, en representaciones discretas y sintácticamente coherentes, es una cognición fría y cerrada en sí misma, etc., supuestos todos ellos compatibles con la lógica computacional subyacente. Pero en nuestra opinión la introducción del contenido como una entidad propia y significativa, no reducible a reglas formales, es incompatible con esos supuestos. Posiblemente esos contenidos sean polivalentes, continuos y hasta cierto punto incoherentes. Además, no terminan en sí mismos, sino que exịsten en la medida en que están dirigidos a un fin, a una acción sobre el mundo. Por ello, pensamos que el pensamiento humano debe estudiarse no sólo en sus aspectos sintácticos o incluso semánticos, sino básicamente en función de su naturaleza pragmática. Sólo de esa forma pueden enterderse las tormentosas relaciones entre forma y contenido en el pensamiento. Esas relaciones se regirían no por un criterio de coherencia formal sino de eficacia pramática. De ser así, estaríamos asistiendo al comienzo de un nuevo romance entre forma y contenido, romance cuyo final no alcanzamos a predecir pero que a buen seguro ayudará a impulsar cambios profundos en las formas y los contenidos de lo que llamamos psicología cognitiva.

\section{Referencias}

ADELSON, J. (1971). The political imagination of the young adolescent Daedalus, 100 (4), 1013-1050. ADI, H; KARPUU, R.; LAWSON, E.A., y PULOS, S.M.(1978) Intellectual development beyond elementary school VI: correlational reasoning. School Science Mathematics, 78, 675-683.

ALOY, L.B., y TABACHNIK, N. (1984). Assesment of covariation by humans and animals: the joint influence of prior expectations and current situacional information. Psychological Review, 91 (1), $112-149$.

asensio, M.; Carretero, M., y Pozo, J.I. (1986). La comprensión de la historia: pensamiento relativista. Cuademos de Pedagogía, 133, 24-27.

AUSUBEL, D.P., y SCHIFF, H.M. (1954). The effect of incidental and experimentally induced experience on the learning of relevant and irrelevant causal relationships by children. Joumal of $G e$ netic Psychology, 84, 109-123.

BindRA, D.; ClaRKE, K.A., y SHULTZ, T.R. (1980) Understanding predictive relations of necessity and sufficiency in formally equivalent scausals and slogicals problems. Journal of Experimental Psychology: General, 109 (4), 422-443.

BRAINE, M.D.S. (1978) On the relation between the natural logic of reasoning and standard logic. Psychological Review, 65, 422-433.

BRAINE, M.D.S., y RUMAIN, B. (1983) Logical reasoning. En J.H. Flavell y E. Markman (Eds.): Handbook of child psychology, Vol. III.: cognitive development (P.H. Mussen, editor general) N. York: Wiley.

CarRETERO, M. (1979) ¿Por qué flotan las cosas? El desartollo del pensamiento hipotético-deductivo y la enseñanza de las ciencias. Infancia y aprendizaje, 8, 7-22.

CARRETERO. M. (1980). Desarrollo intelectual durante la adolescencia: competencia, actuación y diferencias individuales. Infancia y Aprendizaje, 12, 81-98.

Carretero, M. (1984). De la larga distancia que separa la suposición de la certeza. En M. Carretero y J.A. García Madruga (Eds.) Lecturas de psicología del pensamiento. Madrid: Alianza. 
Carretero, M., y Garcia Madruga, J.A. (1984) Psiología del pensamiento: aspectos históricos y metodológicos. En M. Carretero y J.A. García Madruga (Eds.) Lecturas de psicología del pensamiento. Madrid: Alianza.

Carretero, M.; Pozo, J.I., y Asensio, M. (1983). Comprensión de conceptos históticos durante la adolescencia. Infancia y Aprendizaje, 23, $55-74$.

CASE, R. Intellectual development. Birth to adultbood. Orlando: Academic Press.

Chapman, L.J., y Chapman, J.P. (1967). Genesis of popular but erroneous diagnostic observations. Joumal of Abnormal Psychology 72, 193-204.

Chapman, L.J., y Chapman, J.P. (1969). Illusory correlation as an obstacle to the use of valid psychodiagnostic signs. Joumal of Abnormal Psycbology, 74, 271-280.

Cheng, P.W., y Holyonk, K.J. (1985). Pragmatic reasoning schemas. Cognitive Psychology 17, 391.416.

CHI, M.T.H.; GLASER, R., y REES, E. (1982). Expertise in problem solving. En R. Sternberg (Ed.) Advences in the psychology of buman intelligence. Vol. 2. Hillsdale, N.J.: Eralbaum.

ChrostowsKI, J.J., y GRIGGS, R.A. (1985). The effects of problem content, instructions, and verbalization procedure on Wason's selection task. Current Psychological Research \& Reviews, 4 (2), 99-107.

COPPLE, C., y COON, R. (1977). The role of causality in encoding and remebering events as a function of age. Joumal of Genetic Psychology, 130, 129-136.

CROCKER, J. (1981). Judgement of covariation by social perceivers. Psychological Bulletin, 90 (2), $272-292$.

DeIVAL, J.A. (1981). La representación del mundo social en el niño. Infancia y Aprendizaje, 13, 35-66.

EvaNs, J.St.B.T. (1972). On the problems of interpreting reasoning data: logical and psychological approaches. Cognition, 1, 373-384.

Evans, J.St.B.T. (1978). The psychology of deductive reasoning: logic. En A. Burton y J. Radford (eds.): Thinking in perspective. Londres: Methuen. Trad. cast. de J. Fernández: Perspectivas sobre el pensamiento. Madrid: Alhambra, 1984.

EvanS. J.St.B.T. (1982). The psychology of deductive reasoning. Londres: Routledge \& Kegan Paul.

Evans, J.St.B.T. (1984). Heuristic and analitic processes in reasoning. British Jormal of Psychology, $75,451-468$.

ELYON, B-S., y REIF, F. (1984). Effects of knowledge organization on task performance. Cognition and Instruction, 1 (6), 5-44.

FaLmaGnE, R.J. (1975). Overview: reasoning, representation, process and related issues. En R.J. Falmagne (Ed.): Reasoning, representation and process in children and adults. N. York: Wiley.

GARDNER, H. (1985). The mind's new science. N. York: Basic Books.

GrEenwald. A.G. (1975). Consequences of prejudice against the null hypothesis. Psychological Bulletin, 82, 1-20.

GrigGS, R.A. (1983). The role of problem content in the selection task and THOG problem. En J.St.B.T. Evans (Ed.): Thinking and reasoning; psychological approaches. Londres: Routledge \& Kegan Paul.

GrigGs, R.A. (1984) Memory cueing and instructional effects on Wason's selection task. Current Psychological Research \& Reviews, 3 (4), 3-10.

HARVEY, J.H., y WEARY, G. (1984). Current issues in atribution theory and research. Annual Review of Psychology, 35, 427-459.

HENLE, M. (1962). On the relation between logic and thinking. Psychological Review, 69, 366-378. Trad. cast. en J. Delval (Ed.): Investigacines sobre lógica y psicología. Madrid: Alianza, 1977.

INHELDER, B., y PIAGET, J. (1955). De la logique de l'enfant a la logique de l'adolescent. Essai sur la construction des structures opératioires formalles. París: P.U.F. Trad. cast. de M.T. Cevasco: De la lógica del niño a la lógica del adolescente. Buenos Aires: Paidos, 1972.

JenNingS, D.L.; AMABILE, T.M., y Ross, L. (1982). Informal covariation assesment: data-based versus theory-based judgements. En D. Kahneman; P. Slovoc y A. Tversky (Eds.): Judgement under uncertainty: beuristics and biases. Cambridge: Cambridge University Press.

Johnson-LAIRD, P.N.; LegrenZI, P., y Sonino-LegrenZI, M. (1972). Reasoning and a sense of reality. Britsh Jormal of Psychology, 63, 395-400.

JURD, M.F. (1978). Concrete and formal operational thinking in history. En J.A. Keats; K.F. Collis y G.S. Halford (Eds.) Cognitive development. N. York: Wiley.

KARMILOFF-SMITH, A. e INHEIDER, B. (1975). If you to get ahead, get a theory. Cognition, 3, 195-212. Trad. cast. de J.I. Pozo en: Infancia y Aprendizaje, 1981, 13, 67-88.

KeLLEY, H.H. (1973). The process of causal attribution. American Psychologist, 28, 107-128.

Keliey, H.H., y MiCHeLla, J.L. (1980). Attribution theory and research. Annual Review of Psycho$\log y, 31,457-501$.

KUHN, D., y BRANNOCK, J. (1977). Development of the isolation of variables scheme in experimental and anatural experiment contexts. Development Psychology, 13, 9-14.

KuHN, D.; CAPON, N., y CARRETERo, M. (1983). Formal operations and consumer behavior. Investigación inédita. Nueva York: Columbia University.

KuhN, D.; Pennigton, N., y Leadbeater, B. (1983). Adult thinking in developmental perspective: the sample of the juror reasoning. En P. Baltes y O. Brim (Eds.) Life-span developmental 
psychology. Vol. 5. N. York: Academic Press. Trad. cast. no en su totalidad de J.I. Pozo en M. Carretero y J.A. García Madruga (Eds.) Lecturas de psicología del pensamiento. Madrid: AlianZa, 1984.

KUHN, D., y PHELPS, E. (1982). The development of problem solving strategies. En H. Reese (Ed.) Advances in child development and behavior. Vol. 17. N. York: Academic Press.

KULIK, J.A. (1983). Confirmatory attribution and the perpetuation of social beliefs. Joumal of Personality and Social Psychology, 44 (6), 1171-1181.

LAKATOS, I. (1978). The methodology of scientific research programmes-philosophical papers. Volume I. (Ed. de J. Worall y G. Currie). Cambridge: Cambridge University Press. Trad. cast. de J.C. Zapatero: La metodologia de los programas de investigación científica. Madrid: Alianza, 1983.

LAwSSON, E.A. (1983a). The effects of causality, response alternatives and context continuity on hypthesis testing reasoning. Journal of Research in Science Teaching, 20, (4), 297-310.

LAWSON, E.A. (1983b). The acquisition of formal operational schemata during adolescence: the role of the bicondicional. Journal of Research in Science Teaching, 20, (4), 347-356.

LINDSAY, P.H., y NORMAN. D.A. Human information processing. An introduction to cognition. N. York: Academic Press. Trad. cast. de J. Seoane y otros: Introducción a la psicología cognitiva. Madrid: Tecnos, 1983.

LINN, M.C. (1980). Changing the filter on adolescent cognitive behavior: a consideration of context effects. Manuscrito inédito. Berkeley, Universidad de California.

LINN, M.C. (1983). Content, context and process in reasoning during adolescene: selecting a model. Joumal of Early Adolescence, 3, 63-82.

LINN, M.C.; de BENEDICTIS, T., y DEUUCCHI, K. (1982). Adolescent reasoning about advertisements: preliminary investigations. Child Development, S3, 1599-1613.

LINN, M.C.; CIEMENT, C.. y PULOS. S. (1983). It is formal if is not physics? (The influence of content on formal reasoning). Joumal of Research in Science Teaching, 20 (8).

LOU, M.W. (1979). Investigating the development of the logic involved in social/psychological reasoning. Tesis doctoral inédita. Berkeley, Universidad de California.

LURIA, A.R. (1974). Ob istoricheskom razvimii poznavatelnyj protsessov. Moscú: Ed. Nauta. Trad. cast. de Javiovskaya: Los procesos cognitivos. Barcelona: Fontanella, 1980.

MATTHEWS, L., y SANDERS, W. (1984). Effects of causal and noncausal sequences of information on subjetive prediction. Psychological Reports, 54, 211-215.

MAYER, R.E. (1986) Thinking, problem solving, cognition. N. York: Freeman, 1983. Trad. cast. de G. Baravalle: Pensamiento, resolución de problemas y cognición. Barcelona: Paidos.

MILLAR, S. (1982). Studies of the deaf and the blind. En A. Burton (Ed.): The patbology and psycho$\log y$ of cognition. N. York: Methuen.

MOSHMAN, D. (1980). Representation and process in reasoning about logical relationships. The journal of General Psychology, 103, 251-262.

NEWELL, A., y SIMON, H.A. (1972). Human problem solving. Englewood Cliffs, N.J.: Prentice-Hall.

NISBETT, R., y ROSS, L. (1980). Human inference: strategies and shortcomings of social judgement. Englewood Cliffs, N.J.: Prentice-Hall.

PiaGET, J. (1932). Le jugement moral chez l'enfant. París: Alcan. Trad. cast. de N. Vidal: El juicio moral en el niño. Barcelona: Fontanella, 1971.

PIAGET, J. (1970a) Piaget's theory. En P.H. Mussen (Ed.): Carmichael's manual of child psychology. N. York: Wiley. Trad. cast. de M. Serigos en Monografia de Infancia y Aprendizaje, 1981, 2, 13-54.

PIAGET. J. (1970b). L'evolution itellectuelle entre l'adolescence et l'age adulte. Rapport sur le III Congres International FONEME sur la formation humaine a l'age adulte. Milán. Trad. cast. en parte de J.A. Delval en J.A. Delval (Ed.): Lecturas de psicología del niño. Vol. 2. Madrid: Alianza, 1978.

Pozo, J.I. (1985a). El niño y la historia. Madrid: M.E.C.

Pozo, J.I. (1985b). Teorías y reglas de inferencia en la solución de problemas causales. Tesis Doctoral inédita. Universidad Autónoma de Madrid.

Pozo. J.I. (1987). Aprendizaje de la ciencia y pensamiento causal. Madrid: Visor/Aprendizaje.

POZO. J.I., y CARRETERo, M. (1983). El adolescente como historiador. Infancia y Aprendizaje, 23, $75-90$.

Pozo. J.I., y Carretero, M. (1984). ¿Enseñar Historia o contar thistoriass? Otro falso dilema. Cuadermos de Pedagogia, 111, 45-50.

PuLOS, S., y LINN, M. (1981). Generality of controlling of variables scheme in early adolescence. Journal of Early Adolescence, 1 26-37.

READ, S.J. (1983). Once is enough: causal reasoning from a single instance. Journal of Personality and Social Psychology, 45 (2), 323-334.

Ross, L. (1977). The intuitive psychologist and his shortcomings. En L. Berkowitz (Ed.): Advances in Experimental Social Psychology. Vol. 10. N. York: Academic Press.

RUMELHART, D.E. (1980). Schemata: the building blocks of cognition. En R. Spiro, B. Bruce y W. Brewer (Eds.): Theoretical issues in reading comprebension. Hillsdale, N.J.: Erlbaum.

SIMON, D.P., y SIMON, H.A. (1978). Individual differences in solving physics problems. En R. Siegler (Ed.): Children's thinking: what develops? Hillsdale, N.J.: Erlbaum.

SNYDER, M. (1981). Seek and ye shall find: testing hypotheses about other people. En E.T. Higgins; C.P. Herman y M.P. Zanna (Eds.): Social cognition. Hillsdale, N.J.: Erlbaum. 
Trabasso. T.; Secco, T., y VAN den Broek. P. (1984) Causal cohesion and story coherence. En $\mathrm{H}$. Mandl, N.L. Stein y T. Trabasso (Eds.): Leaming and comprebension from text. Hillsdale, N.J.: Erlbaum.

TVERSKY, A., y KAHNEMAN, D. (1984). Judgement under uncertainty: heuristics and biases. Science, 1974, 185, 1124-1131. Trad. cast. de J.I. Pozo en M. Carretero y J.A. García Madruga (Eds.): Lecturas de psicología del pensamiento. Madrid: Alianza.

TVERSKY, A., y KAHNEMAN, D. (1982). Causal schemas in judgement under uncertainty. En D. Kahneman; P. Slovic y A. Tversky (Eds.): Judgment under uncertainty: heuristics and biases. Cambridge: Cambridge University Press.

VALENTINE, E.R. (1985). The effects of instructions on performance in the Wason selection task. Current Psychological Research \& Reviews, 4, 214-223.

VEGA, M. (1981). Una exploración de los metapostulados de la psicología contemporanea: el logicismo. Análisis y Modificación de Conducta, 16, 345-376.

WASON, P.C. (1977). The theory of formal operations: a critique. En B. Geber (Ed.): Piaget and knowing. Londres: Routledge \& Kegan Paul. Trad. cast. de M.M. Prelooker: Piaget y conocimiento. Barcelona: Paidos, 1980.

WASON, P.C. (1983). Realism and rationality in the selection task. En J.St.B.T. Evans (Ed.): Thinking and reasoning. Psychological approaches. Londres: Routledge \& Kegan Paul. Trad. cast. de E. Martín en M. Carretero y J.A. García Madruga (Eds.): Lecturas de psicología del pensamiento. Madrid: Alianza, 1984.

WasON, P.C., y Evans, J.St.B.T. (1975) Dual processes in reasoning? Cognition, 3, 141-154.

WASON, P.C., y GREEN, D.W. (1984). Reasoning and mental representation. The Quarterly of Experimental Psychology, 36A, 597-610.

WASON. P.C., Y JOHNSON.LAIRD, P.N. (1972). Psychology of reasoning: structure and content. Londres: Batsford Ltd. Trad. cast. de J.A. Delval: Psicologia del razonamiento. Madrid: Debate, 1981.

Whiteman, M. (1967) Children's conceptions of psychological causality. Child Development, 38, 143-155.

WhITEMAN, M. (1970). The development of conceptions of psychological causality. En J. Hellmuth (Ed.): Cognitive studies. Vol. 1. N. York: Brunner/Mazel.

WOODWORTH, R.S., y SELS, S.B. (1935). An atmosphere effect in formal syllogistic reasoning. Journal of Experimental Psychology, 18, 451-160. Trad. cast. de V. Demonte en J.A. Delval (Ed.): Investigaciones sobre lógica y psicología. Madrid: Alianza, 1977.

WRIGHT, J.C., y MURPHY, G.L. (1984). The utility of theories in intuitive statistics: the robutness of theory-based judgement. Joumal of Experimental Psychology: General, 113, (2), 301-322.

YACHANIN, S.A. (1986). Facilitation in Wason's selection task: content and instructions. Current Psychological Research \& Reviews, 5 (1), 20-29.

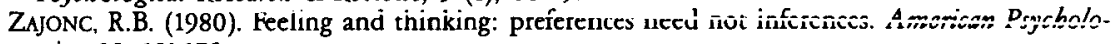
gist, 35, 151-175. 